\title{
Measurement of electromagnetic interference in time-domain
}

\author{
S. Braun, A. Frech, and P. Russer \\ Lehrstuhl für Hochfrequenztechnik, Arcisstraße 21, 80333 München, Germany
}

\begin{abstract}
Time-domain EMI measurement systems allow measurement time to be reduced by several orders of magnitude. In this paper a novel real-time operating time-domain EMI measurement system is presented. By the use of several analog-to-digital converters the dynamic range requested by the international EMC standards is achieved. A real-time operating digital signal processing unit is presented. The frequency band that is investigated is subdivided into several sub-bands. A novel implementation of the $9 \mathrm{kHz}$ IF filter for the frequency $150 \mathrm{kHz}$ to $1 \mathrm{GHz}$ is presented. By this way the measurement time has been reduced by a factor of 8000 in comparison to conventional EMI receivers. During emission measurements performed with a modelled IF-bandwidth of $9 \mathrm{kHz}$ the noise floor is decreased to $-19 \mathrm{~dB} \mu \mathrm{V}$ in the average detector mode by the implemented low noise power splitter. Measurements have been performed with the improved measurement system in the frequency range $30 \mathrm{MHz}-1 \mathrm{GHz}$.
\end{abstract}

\section{Introduction}

Traditionally emission measurements are carried out by EMI receivers operating in frequency domain. During the last years we have performed research on time-domain EMI (TDEMI) measurement systems. It has been shown that by a multiresolution TDEMI measurement system sufficient high dynamic range is achieved (Braun et al., 2005; Braun and Russer, 2005). By real-time implementation of the digital signal processing on field programmable gate arrays (FPGAs) the measurement time for a single scan has been reduced by a factor of 2000 (Braun et al., 2006) in the CISPR Band C, D. By this way continuous processing has been implemented as required by CISPR 16-1-1 (2006).

For measurements that have very tight requirements, e.g. measurements where the compliance limit is at $0 \mathrm{~dB} \mu \mathrm{V}$, a measurement system with a low noise figure and an IFbandwidth of $9 \mathrm{kHz}$ is required (General Motors Engineering Standards, 2006; DaimlerChrysler, 2006). For such measurements the number of frequency bins increases up to 190000 ,

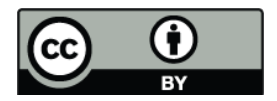

Correspondence to: A. Frech (frech@tum.de) while the measurement system must provide a low noise figure. Such measurements take a factor of more than 10 longer than measurements with an IF-bandwidth of $120 \mathrm{kHz}$. For some products the required test time even exceeds the product life time. EMI receivers that have no preamplifier do not have enough sensitivity to perform such measurements. Often low-noise preamplifiers are used in front of an EMI receiver to reduce the overall noise figure of the measurement setup. But by such a setup the dynamic range is limited by the $1 \mathrm{~dB}$ compression point of the preamplifier. The result is the clipping of signals that require a high dynamic range, e.g. transient signals. Such a setup does not provide enough overload protection.

In the following an improved real-time TDEMI measurement system is presented that allows to calculate via the short-time fast Fourier transform (STFFT) a number of 8192 frequency bins with a modelled IF-bandwidth of $9 \mathrm{kHz}$ simultaneously. During the digital signal processing a numerical oscillator is used to increase the frequency resolution and reduce the picket fence effect. By this way the measurement time has been reduced by a factor of about 8000 for a single scan in comparison to a conventional system. By a low-noise implementation of the floating point analog-to-digital conversion the noise figure has been decreased to about $7.5 \mathrm{~dB}$.

\section{Time-domain EMI measurement system}

By a TDEMI measurement system the input signal is digitized by an analog-to-digital converter (ADC) system. Spectral estimation is performed by the fast Fourier transform (FFT). The block diagram of a multiresolution TDEMI measurement system is shown in Fig. 1. The EMI signal is received via an ultra broadband antenna (Rohde \& Schwarz, 2001). By a multiresolution analog-to-digital converter (ADC) unit a floating point analog-to-digital conversion is performed (Braun and Russer, 2005). By noise optimization of the power splitter and limiter circuits used for splitting and scaling of the input signal for the ADCs the noise figure is reduced to $7.5 \mathrm{~dB}$, while a reflection coefficient below $-15 \mathrm{~dB}$ has been achieved. By this way the system can digitize small signals and short pulses up to an amplitude range of about $6 \mathrm{~V}$ with an average noise floor of about $-9 \mathrm{~dB} \mu \mathrm{V}$ with a

Published by Copernicus Publications on behalf of the URSI Landesausschuss in der Bundesrepublik Deutschland e.V. 


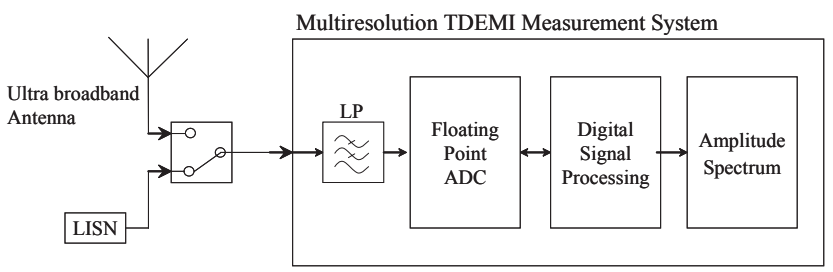

Fig. 1. Multiresolution time domain EMI measurement system.

modelled IF filter bandwidth of $120 \mathrm{kHz}$. The measured and digitized EMI signal is processed digitally and the amplitude spectrum is displayed. The operation of a real-time multiresolution TDEMI measurement system has already been presented in (Braun et al., 2006).

The frequency range from $\mathrm{DC}$ to $1 \mathrm{GHz}$ is subdivided into eight bands that are measured sequentially. The signal is digitally downconverted with a bandwidth of $125 \mathrm{MHz}$ and is processed by a real-time operating short-time fast Fourier transform. The real-time operating short-time fast Fourier transform simultaneously processes 8192 frequency bins. The IF filter with a bandwidth of $9 \mathrm{kHz}$ is implemented by a Gaussian window function. The simulation of the detector modes, e.g. the peak detector, is performed at 8192 frequency bins simultaneously. By this way the measurement time can be reduced by a factor of about 8000 in comparison to a measurement performed with a conventional EMI receiver.

\subsection{Fast Fourier Transform}

Digital spectral estimation is performed via the Discrete Fourier Transform (DFT). FFT algorithms are applied exploiting symmetry and repetition properties of the DFT. The DFT formulation considers periodic repetition of the timedomain signal and is given as follows:

$X[k]=\sum_{n=0}^{N-1} x[n] e^{\frac{-j 2 \pi k n}{N}}$

\subsection{Short-time Fast Fourier Transform}

By the STFFT a spectrogram is calculated. This spectrogram shows a discretisation in frequency and time. The resolution in frequency is described by the bin width $\triangle f$. The resolution in time is described by a time step $T_{S B B}$ corresponding to the increment used in the STFFT (Braun et al., 2004). The inverse of the time step is the baseband sampling frequency $f_{s b b}$ which is used to simulate the peak, quasi-peak, average, and rms detector mode. The spectrogram calculated by the STFFT is given by

$X[t, k]=\sum_{n=0}^{N-1} x[n-t] w[n] e^{\frac{-j 2 \pi k n}{N}}$

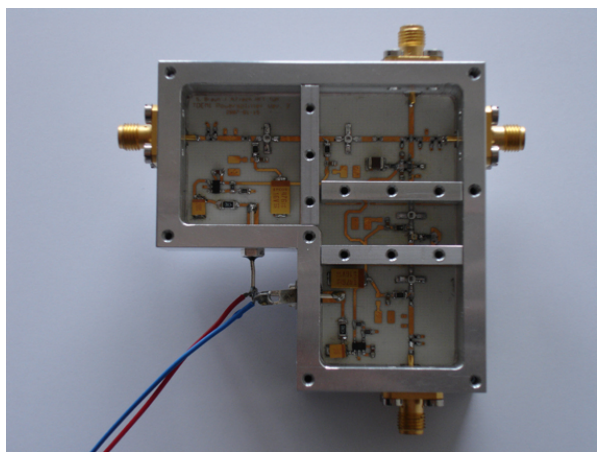

Fig. 2. Power splitter.

where $w[n]$ is a Gaussian window function that models the IF filter of an EMI receiver.

\subsection{Noise behavior}

The TDEMI measurement system shows a noise figure of about $7.6 \mathrm{~dB}$. With a modelled IF-bandwidth of $120 \mathrm{kHz}$ an equivalent noise bandwidth of $90.3 \mathrm{kHz}$ is obtained. Thus for the TDEMI measurement system an average noise floor of about $-10 \mathrm{~dB} \mu \mathrm{V}$ is calculated. For a $9 \mathrm{kHz}$ IF filter an average noise floor of about $-21 \mathrm{~dB} \mu \mathrm{V}$ is obtained.

\subsection{Hardware implementation}

\subsubsection{Low-noise power splitter}

The photo of the realized power splitter is shown in Fig. 2. The received signal is distributed by an asymmetric powersplitter into three channels with different sensitivity. The first channel exhibits an amplification of $24.5 \mathrm{~dB}$ to digitize the amplitude range up to $30 \mathrm{mV}$. It uses a low-noise lowreflection limiter circuit followed by a series of low-noise amplifiers. The thermal noise of the broadband amplifiers has to be attenuated in order to suppress aliasing effects. Such aliasing of the thermal noise leads to a degradation of the noise performance of the system. Thus a slight attenuation is performed above $1 \mathrm{GHz}$. The second channel exhibits an attenuation of $2 \mathrm{~dB}$. By this way the amplitude range up to $630 \mathrm{mV}$ is digitized. Channel three shows an attenuation of $25.5 \mathrm{~dB}$. By this way signals up to $9 \mathrm{~V}$ can theoretically be digitized. However above $6 \mathrm{~V}$ the performance degrades. Additionally all channels feature limiter diodes to protect the input ports of the analog-to-digital converters.

The noise figure has been characterized by an HP8970B noise figure meter. The result is shown in Fig. 3. The noise figure in the range $100 \mathrm{MHz}-1 \mathrm{GHz}$ is about $7.5 \mathrm{~dB}$. For lower frequencies the noise figure is degraded by shot noise and 1/f noise (Meinke and Gundlach, 1986). 


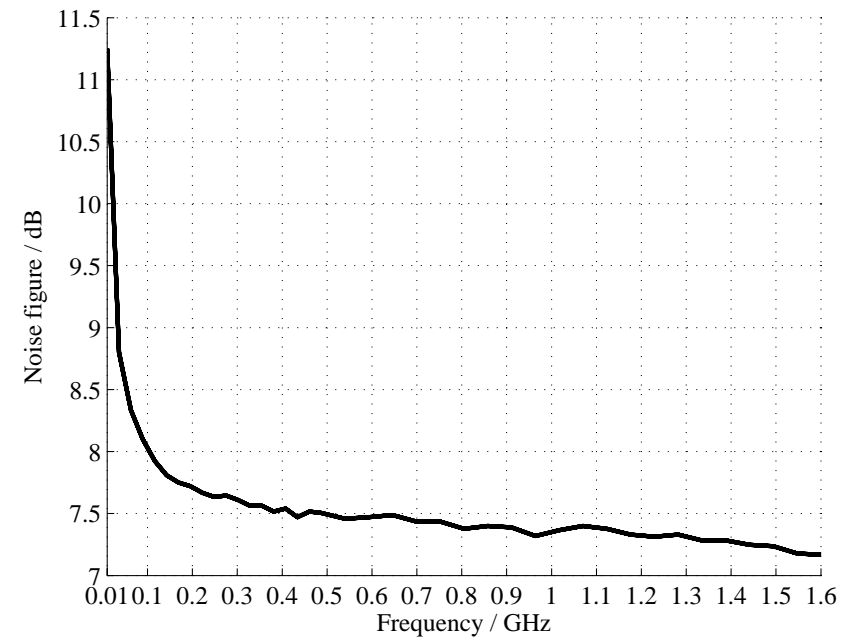

Fig. 3. Noise figure.

\subsubsection{Analog-to-digital converter system}

The three ADCs are clocked by a common clock signal. The input of each ADC is connected to the output of the power splitter limiter circuit. The outputs of the ADCs are connected to the first field programmable gate array, where the EMI signal is reconstructed with a high resolution. The realtime signal processing is performed partially on the first and second FPGA.

\section{Measurements}

The input of the TDEMI measurement system has been connected to a $50 \Omega$ resistor. The indicated noise floor for an IF-bandwidth of $120 \mathrm{kHz}$ is below $-7 \mathrm{~dB} \mu \mathrm{V}$. One spurious signal originating from the ADC can be observed at fs $/ 4$. For an accurate measurement at those frequencies the sampling frequency can be changed to perform the measurement at the frequencies around $\mathrm{fs} / 4$.

An emission measurement was performed with a modelled IF-bandwidth of $9 \mathrm{kHz}$. The peak detector mode was selected. The scan with the EMI receiver took several minutes. With the real-time TDEMI measurement system the scan took about $10 \mathrm{~ms}$. The result is shown in Fig. 4. The deviations are below $3 \mathrm{~dB}$.

\section{Conclusion}

The noise figure has been improved to $7.5 \mathrm{~dB}$ while a reflection coefficient below $-15 \mathrm{~dB}$ has been achieved. The system can capture single pulses up to an amplitude of $6 \mathrm{~V}$ without an additional attenuator. Thus measurements can be performed with a very low noise figure while providing a sufficient high overload protection. A typical application is the measurement of automotive components and systems.

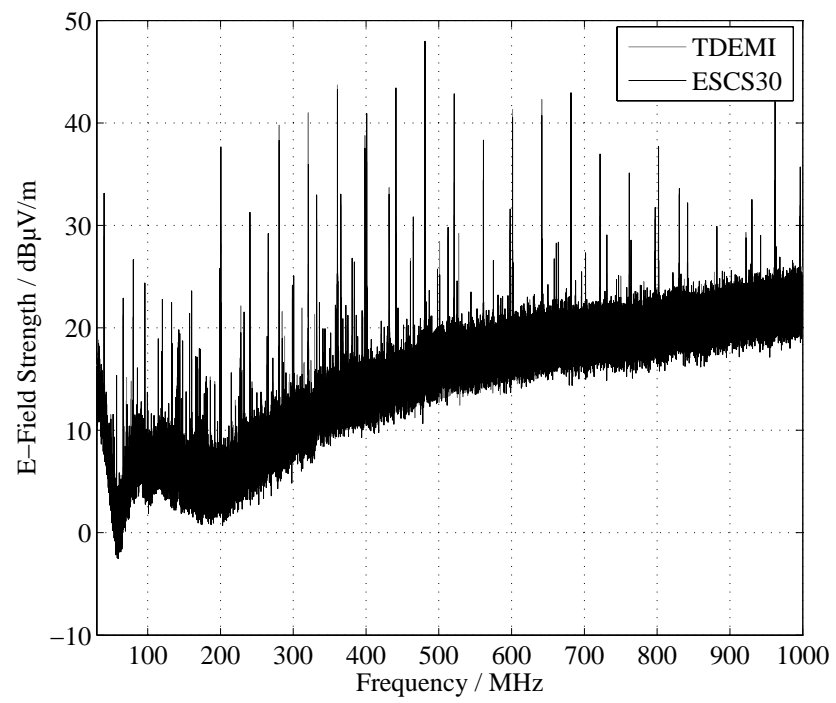

Fig. 4. Emission measurement of a laptop.

Acknowledgements. This work was supported by the Deutsche Forschungsgemeinschaft.

\section{References}

Braun, S. and Russer, P.: A Low-Noise Multiresolution HighDynamic Ultra-Broad-Band Time-Domain EMI Measurement System, IEEE Transactions on Microwave Theory and Techniques, 53, 3354-3363, 2005.

Braun, S., Krug, F., and Russer, P.: A Novel Automatic Digital Quasi-Peak Detector for a Time Domain Measurement System, in: 2004 IEEE International Symposium On Electromagnetic Compatibility Digest, August 9-14, Santa Clara, USA, vol. 3, pp. 919-924, 2004.

Braun, S., Aidam, M., and Russer, P.: Development of a Multiresolution Time-Domain EMI Measurement System that Fulfils CISPR 16-1, in: 2005 IEEE International Symposium On Electromagnetic Compatibility, Chicago, USA, pp. 388-393, 2005.

Braun, S., Al-Qedra, M., and Russer, P.: A novel Realtime Time-domain EMI Measurement System based on Field Programmable Gate Arrays, in: 17th International Zurich Symposium on Electromagnetic Compatibility, Digest, pp. 501-504, Singapore, 2006.

CISPR 16-1-1: Specification for radio disturbance and immunity measuring apparatus and methods Part 1-1: Radio disturbance and immunity measuring apparatus - Measuring apparatus, International Electrotechnical Commission, 2006.

DaimlerChrysler: Joint Engineering Standard DC-11223, DaimlerChrysler, 2006.

General Motors Engineering Standards: General Specification for Electrical/Electronic Components and Subsystems, Electromagnetic Compatibility, GMW3097, GM, 2006.

Meinke, F. and Gundlach, H.: Taschenbuch der Hochfrequenztechnik, ISBN 3-540-15393-4, Springer Verlag, 1986.

Rohde \& Schwarz: Ultra Broadband Antenna HL562 ULTRALOG, Data sheet, 2001. 